\title{
Current Progresses in the Development of Biosensors for the Diagnosis of Neglected Tropical Diseases
}

\author{
Pedro Augusto de Freitas Borges, Wagner Augusto Fiel, Vitor Azevedo Vasconcellos ${ }^{\circledR}$, Ricardo \\ Adriano Dorledo de Faria*
}

Department of Chemical Engineering, Federal University of Minas Gerais, Belo Horizonte, MG, Brazil

E-mail: ricardo.adriano08@hotmail.com

Received: 27 April 2020; Revised: 15 June 2020; Accepted: 29 June 2020

\begin{abstract}
The World Health Organization classifies neglected tropical diseases (NTDs) in order to highlight the impact of these diseases on public health and as a tool to eradicate them. Due to its risk to human health, especially in the last decade, several publications have been dedicated to the proposal of alternative diagnosis for NTDs. The rapid detection of these diseases is essential for the early initiation of treatment and, thus, allows the reduction of mortality and improves the quality of life of patients. However, the conventional techniques used to detect NTDs are expensive, time-consuming and require more sophisticated equipment in the laboratory, which hinders analyzes to be performed in situ. In light of this context, researchers have been working on the application of biosensors to overcome the current problems. The results found have been promising, as the biosensors exhibited low detection limits with high selectivity and the possibility of performance in miniaturized/portable systems. This technology combines the specificity of biological probes with the sensitivity and stability of several groups of transducers (mainly electrochemical and optical ones). In the present manuscript, we reviewed the recent advances in the field of biosensors to detect some of the main pathogens causing NTDs worldwide (Zika, dengue, schistosomiasis, malaria, snakebite envenoming, leishmaniasis and Chagas disease).
\end{abstract}

Keywords: biosensor, neglected tropical diseases, diagnosis, transducers

\section{Introduction}

Summarized by the World Health Organization (WHO), the neglected tropical diseases (NTDs) comprise a current list of the following 20 diseases that strongly affect approximately one billion people in low-income countries mainly due to the poor health/economic conditions of these regions $[1,2]$ :

- Buruli ulcer;

- Chagas disease;

- Dengue and Chikungunya;

- Dracunculiasis (guinea-worm disease);

- Echinococcosis;

- Foodborne trematodiases;

- Human African trypanosomiasis (sleeping sickness);

Copyright (C2020 Ricardo Adriano Dorledo de Faria, et al

DOI: https://doi.org/10.37256/sbe.112021402

This is an open-access article distributed under a CC BY license

(Creative Commons Attribution 4.0 International License)

https://creativecommons.org/licenses/by/4.0/ 
- Leishmaniasis;

- Leprosy (Hansen's disease);

- Lymphatic filariasis;

- Mycetoma, chromoblastomycosis and other deep mycoses;

- Onchocerciasis (river blindness);

- Rabies;

- Scabies and other ectoparasites;

- Schistosomiasis;

- Soil-transmitted helminthiases;

- Snakebite envenoming;

- Taeniasis/Cysticercosis;

- Trachoma;

- Yaws (Endemic treponematoses).

According to WHO, diseases that affect the poorest population are called "poverty diseases" and can be divided into two types: the first one includes diseases such as tuberculosis and AIDS, which received greater attention from the scientific community on forms of treatment and eradication due to their high potential impact. The second type includes neglected diseases (such as dengue, zika, Chagas disease, leishmaniasis, among others) which have lower visibility, even though some of them have existed for decades and pose significant risk to humans' health [3].

Prevailing in needy regions, specifically in the tropics, where the warm and humid weather conditions favor the vectors development, global efforts have been directed towards the eradication of these diseases [4]. However, to convert this effort in efficient results, it is equally necessary to develop accurate technologies on the detection and diagnosis of NTDs. Thus, the management of appropriate drugs in the patient can be performed at a very primary stage in order to avoid the pathogenic effects of the biological vectors.

In light of this context, the use of biosensors appears as an alternative to the traditional techniques due to their lower cost, lower time-to-perform, possibility of miniaturization (which allows the fabrication of portable devices) and the low limit of detection (LOD) [5]. Consequently and taking advantage of the various possible biological probes, many researchers have dedicated their expertise to fabricate versatile biosensors capable of recognizing different analytes, achieving high levels of selectivity, sensitivity and with easier applicability in places where these diseases typically ocurr [6]. The biosensing mechanism of these devices consists on the biochemical interactions between a biological element attached to a transducer substrate and an analyte of interest from a certain bulk solution. This reaction leads to the alteration of a transduction property of the sensor (e.g. conductivity, temperature, absorbance, etc.) and this signal variation is proportional to the analyte concentration [7]. Figure 1 illustrates the main components of a biosensor. Depending on the kind of monitored output signal, the biosensor can be classified as thermal, optical, electrochemical, piezoelectric, acoustic, etc.. In our previous publications, we have demonstrated the versatility, high sensitivity and selectivity of biosensors in detecting very distinct molecules, such as some contaminating pharmaceuticals present in aquatic environments [8] and polycyclic aromatic hydrocarbons [5], both cases related to substances of public concern.

The successful performance of a biosensor also relies on the use of an appropriate transducer substrate. Shetti et al. [9] highlighted the importance of the nanotechnology on the development of analytical platforms for clinical diagnostics and discussed the promising properties of $\mathrm{ZnO}$-based electrodes for constructing electrochemical (bio)sensors. According to the authors, the noncentrosymmetric feature of the $\mathrm{Zn}^{2+} / \mathrm{O}^{2-}$ structure makes this oxide especially amenable for piezoelectric sensors. In another work, Shetti et al. [10] evidenced $\mathrm{TiO}_{2}$-based devices applicable in the field of healthcare analysis. The researchers pointed out that this material possesses important characteristics for the sensing applications, such as the photo-corrosion resistance, biocompatibility, non-toxicity as well as the low cost associated to the easiness of large-scale production. Considering these prominent features, Faria et al. [11] studied the electrochemical behavior of a $\mathrm{TiO}_{2}$ sol-gel coating and used this material as a transducer substrate in an impedimetric immunosensor for the diagnosis of an important NTD: snake envenoming. In their findings, $\mathrm{TiO}_{2}$ was obtained as an n-type semiconductor that, after functionalization with antibodies produced against Bothrops venom, was capable to detect the analyte at a concentration of $20 \mu \mathrm{g} \cdot \mathrm{mL}^{-1}$ in less than one hour.

In the present manuscript, we briefly reviewed the literature on the latest advances in the field of biosensors applied 
to the diagnosis of NTDs worldwide in comparison to the traditional assays. Herein, aspects related to the transduction techniques, limitation of conventional techniques, sensitivity and selectivity of recent biosensors were raised with respect to the most important NTDs. Thus, the objective of this research was to introduce a brief description on the theory of biosensors as well as to contribute to the visibility of the NTDs, once their current diagnoses still represent an important drawback to their treatment and, consequently, pose risk to the patients' lives.

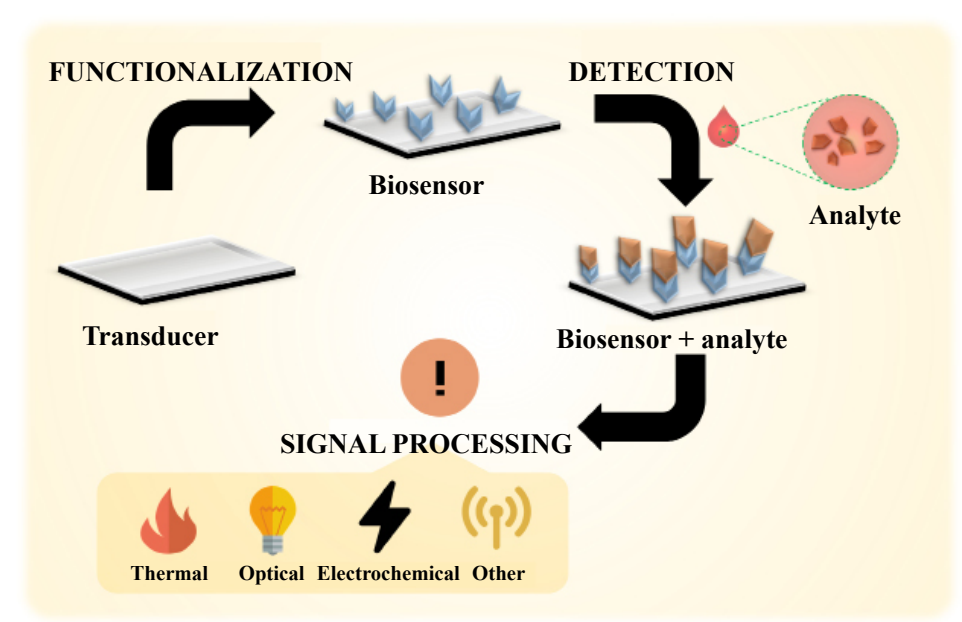

Figure 1. Main components of a biosensor and its interaction with the target analyte altering the measurable output signal

\section{Main NTDs and diagnosis}

The NTDs are common in regions where the levels of hygiene, sanitation and access to health are precarious. In this sense, because of the precariousness of medical assistance in these places, the utilization of rudimentary methods for diagnosing some diseases is still common and the classic assays have failed to provide a rapid response to the advance of these diseases [5]. Once approximately $40 \%$ of the world population inhabits between the Tropic of Cancer and the Tropic of Capricorn, the possibility of contracting NTDs is a real global issue [8]. According to WHO, the impact of NTDs is related not only to the favorable climatic conditions of the tropics to vectors growth and development, but it is also associated to the slow and inefficient diagnoses. This issue happens due to the precarious public health programs in low-income countries as well as to the phenomenon of accelerated unplanned urbanization in overpopulated regions with poor access to basic sanitation [12].

In the present work, recent trends in the area of biosensors for NTDs detection were categorized according to the pathology to which they refer.

\subsection{Zika}

Zika virus causes one of the NTDs that poses major risk to public health. Although the first cases indicating the contamination by the virus are recent in some regions of the planet, the disease causes several health complications, such as some neurologic disturbs (correlation with the Guillain-Barré syndrome for instance), microcephaly, acute fever, adverse fetal outcomes, etc. [13]. Petersen et al. [13] reported that the first isolation of Zika virus dates from 1947 in Uganda. However, the global concern with respect to the impact of the disease came only from 2007, when approximately 5000 people out of 6700 habitants of the State of Yap in the Federated States of Micronesia were diagnosed with Zika virus. In the Americas, only in March 2015 the first case of Zika virus was reported and it occurred in Bahia, Brazil.

The Zika virus cycle transmission occurs from infected Aedes Aegypt mosquitoes to humans. In infected people, the virus is found in blood, urine, semen, saliva and other body fluids. Thus, the disease can be transmitted through sexual contact, blood transfusion, intrauterine/intrapartum via and laboratory exposure. Currently, there is still no available 
drug or vaccine treatment. Some medicines, though, can be useful for relieving fever, pain and other side effects. In this sense and in order to take some cares about the disease (regarding the transmission for example), it is crucial to patients to receive an accurate and rapid diagnosis $[14,15]$.

Currently, the diagnosis of the disease is still limited and somehow fragile. An initial challenge is the fact that Zika, dengue and chikungunya viruses share similar general symptoms, which represents a difficulty to differentiate the three diseases. Moreover, laboratorial tests performed with blood fluids require very specialized equipment and skilled technicians, which also implicates in very expensive methods of detection and limits it as a global tool for the disease diagnosis [16]. Prior to 2016, the United States of America were the only country in which the diagnostic method for Zika virus was performed in the world. Although after the global outbreak in the past few years, some manufacturers were allowed to apply for an emergency authorization to carry out their test assays for diagnosing the disease. Despite the availability of these new temporarily authorized assays, the most needy regions in which Zika virus is endemic still remains uncovered by the reliable diagnoses [17]. In this context, Theel and Hata [17] highlighted the urgent necessity for point-of-care devices that meet the WHO criteria for diagnosing Zika virus, which are related to the sensitivity, affordability, selectivity, timely performance, robustness, deliverability, to be user-friendly and equipment-free.

As a response to the challenges linked to the conventional diagnostic of Zika virus (polymerase chain reactionPCR) [18], Kaushik et al. [6] developed an electrochemical immunosensor by means of the immobilization of a specific Zika virus-envelop protein antibody on a monolayer of dithiobis (succinimidyl propionate) (DTSP) deposited on a gold interdigitated microelectrode. The authors performed the technique of Electrochemical Impedance Spectroscopy (EIS) to monitor the charge transfer resistance $\left(\mathrm{R}_{\mathrm{ct}}\right)$ resultant from the interaction between the immobilized antibody and the Zika antigen. Due to its insulating behavior, the formed immunocomplex hindered the electronic and charge transfer processes at the interface electrode/electrolyte. The device presented a detecting range from $10 \mathrm{pM}$ to $1 \mathrm{nM}$ and a sensitivity of $12 \mathrm{k} \Omega \cdot \mathrm{M}^{-1}$. Besides, the proposed biosensor exhibited high selectivity towards the analyte of interest, with the generation of small output signal when exposed to other viruses of chikungunya and Nile West at $50 \mathrm{pM}$ (variation of $\mathrm{R}_{\mathrm{ct}}$ equal to $2 \%$ and $3 \%$, respectively). The authors mentioned that one of the main advantages of the proposed sensor was the capability of the device to transfer the results to a smartphone remotely, which could facilitates the detection of the disease even in endemic regions and the global monitoring of supporting data.

In the same context, Priye et al. [16] reported the fabrication of a point-of-care smartphone-based sensor that was capable to provide a sensible optical response towards Zika virus. According to their report, the achievements of this novel technology included the unambiguous detection of the Zika virus directly in body fluid samples, with no crossreactivity observed when exposing the device to other similar viruses.

In 2018, Tancharoen et al. [19] published an manuscript reporting the decoration of surface imprinted polymers with graphene oxide. Briefly, the fabrication of the sensitive transducer substrate consisted in preparing a prepolymer gel, mixing the graphene oxide, attaching the obtained composite on a gold electrode and dropping a solution of Zika virus on the material. After exposing the film containing the virus to UV light for $3 \mathrm{~h}$, the electrode was kept at $65^{\circ} \mathrm{C}$ for $15 \mathrm{~h}$ in an oven to complete the polymerization step. Finally, the material was treated with an acid solution and deionized water at $50^{\circ} \mathrm{C}$ for 30 minutes to obtain the Zika virus template. Then, the detection tests were performed and exhibited good sensitivity through both EIS and Cyclic Voltammetry (CV) measurements. In EIS, the authors monitored the variation of $\mathrm{R}_{\mathrm{ct}}$ as a response to the virus concentration (in both PBS and serum). In CV results, the variations in the current of the redox peaks corroborated the impedimetric detection, revealing there was a liner correlation between the electrochemical signal and the analyte concentration. The authors reported a LOD of $2 \times 10^{-4}$ plaque forming units (PFU) per milliliter in PBS and $2 \times 10^{-3} \mathrm{PFU} \cdot \mathrm{mL}^{-1}$ in serum.

\subsection{Dengue}

Similarly to Zika disease, the Aedes Aegypt mosquito is the vector related to the transmission of dengue to humans. There are four serotypes of the genus Flavivirus related to the RNA virus, which are named DENV-1, DENV-2, DENV3 and DENV-4 [20]. Depending on the incidence of the illness (if it is the first or second infection), the patient can be a victim of dengue fever or dengue hemorrhagic fever.

To diagnose dengue, the assay consists in creating a cDNA copy of the genome RNA, following by an amplification mediated by Taq polymerase [21]. Despite very accurate, this technique is very time-consuming, taking a minimum of 
30 hours to provide the result. In this context, most of the dengue cases are diagnosed through the observation of the patient's symptoms, method that can lead to the misinterpretation and false-negative results [22].

Recent developments in the field of biosensing technologies have purposed new possibilities to dengue diagnosis. Kim et al. [20] developed a selective biosensor for detecting the dengue virus using a peptide capable to detect the nonstructural glycoprotein biomarker 1 (NS1). These receptors were attached to a previously treated gold electrode. The researchers isolated the genetically modified peptides and used these selective molecules to originate five receptors: DGV BP1, DGV BP2, DGV BP3, DGV BP4, and DGV BP5. As transduction techniques, the authors performed EIS, Square-Wave Voltammetry (SWV) and Circular Dichroism (CD), from which they assessed the peptides and elected the DGV BP1 as the most sensitive to the target antigen. The developed biosensor presented a LOD equal to $1.49 \mu \mathrm{g} \cdot \mathrm{mL}^{-1}$, stability of $10 \mathrm{~h}$ in solution with low signal interference and high selectivity towards the analyte of interest, being capable not only to detect but also to quantify the concentration of the virus in plasma samples [20].

Under the same context, Jahanshahi et al. [23] also worked on the dengue diagnosis. In their work, the authors reported the fabrication of a biosensor based on the surface plasmon resonance (SPR) technique. In few minutes of exposure, the biosensor was capable to detect immunoglobulin $\mathrm{M}(\operatorname{IgM})$ as an indicative of dengue infection in a small volume of blood sample $(1 \mu \mathrm{L})$. The main advantages concerning this optical system was the detection speed (10 minutes), the easiness of application as a point-of-care technique, the high sensitivity (83\%-93\%) and the total selectivity.

Basso et al. [24] described the development of a colorimetric dengue biosensor using a hybrid nanomaterial consisting of magnetic $\gamma-\mathrm{Fe}_{2} \mathrm{O}_{3}$ nanoparticles, gold nanoparticles and aptamers (with the sequence 5'-SH/-CCCGCAC CGGGCAGGACGTCCGGGGTCCTCGGGGGG CGGG-3' specific for the recognition of the four dengue serotypes). After illuminating the biosensor with unpolarized light, the authors observed a displacement of the wavelength and a decrease in the intensity of the absorbance peak because of the aptamer-virus conjugation. Furthermore, the hybridization of the nanomaterial with the aptamers changed the color of the analyte solution from dark purple to green. In order to assess the selectivity of the proposed device, the researchers tested the biosensor against Zika virus and Yellow fever virus because both present structure similar to the dengue virus, which did not change the solutions colors, indicating there was no aptamer-virus binding.

Reaffirming the importance and need for an early diagnosis of dengue, Zainuddin et al. [25] proposed the construction of an integrated system for the ultrasensitive detection of dengue virus measuring both mass and impedance as transduction signals. Named electrochemical quartz crystal microbalance biosensor, this kind of device combines the high sensitivity of piezoelectric measurements with the high selectivity and linearity provided by electrochemical techniques (besides serving as its own cross-validation method). In the presence of $\left[\mathrm{Fe}(\mathrm{CN})_{6}\right]^{3 / 4-}$ redox probe, the $\mathrm{R}_{\mathrm{ct}}$ of the integrated biosensor increased due to the interaction of the sensitive layer containing anti-NS1 IgG antibodies and the NS1 antigen. At the same time, the analyte caused a decrease in the variation of the system frequency as expected from the Sauerbrey equation (Equation 1), which rules the dependence of the resonance frequency of the quartz crystal microbalance and its mass [26]. In this equation, $f_{0}$ is the natural frequency of the crystal resonator, $\Delta f$ is the variation of the crystal frequency due to the variation of mass $(\Delta \mathrm{m})$ on its surface, $A_{q}$ is the surface area, $\mu_{q}$ is the shear modulus and $\rho_{q}$ is the density.

$$
\Delta f=-\frac{2 f_{0}^{2}}{A_{q} \sqrt{\mu_{q} \rho_{q}}} \Delta m
$$

\subsection{Schistosomiasis}

The contamination by Schistosoma mansoni is one of the NTDs with the highest case fatality rates. Schistosomiasis presents high incidence mainly in the poorer regions of the planet, affecting approximately 260 million people [27, 28]. Neuroschistosomiasis, the most aggressive form of the disease, is characterized by the contamination of the central nervous system. Due to its immense risk to the public health, the fast and precise diagnosis takes an important place in the monitoring of the disease. The WHO recommends the Kato-Katz (KK) method as the standard technique for detecting the infection by $S$. mansoni. This method reveals the quantity of parasite eggs as an indirect parameter linked to the host helminth burden [29]. Despite the relative low cost and the high selectivity, the KK assay finds its main 
challenge in the sensitivity aspect because it strictly depends on the amount of available parasite eggs [30]. Wu et al. [30] mention that the widely spread immunological techniques used for diagnosing this illness are limited in low-intensity transmission areas, because of the difficulty to distinguish current and past infections (because raised antibodies remain for longtime periods after the first infection) and due to the high cost of the necessary laboratorial apparatus and chemicals.

To overcome these challenges, Santos et al. [31] developed a label-free electrochemical biosensor based on the combination of self-assembled 3-mercaptopropyltrimethoxysilane (MPTS) layers, gold nanoparticles transducer matrix and DNA as a biological probe. The variation of the genosensor's impedance by means of the $\mathrm{R}_{\mathrm{ct}}$ variation was inspected to infer about the $S$. mansoni detection. The results revealed a LOD of $0.6 \mathrm{pg} \cdot \mu \mathrm{L}^{-1}$ and the authors proved that this performance was in good agreement with a conventional PCR method and that the sensor was capable to provide a sensitive analytical response even when the analyte was diluted in some body fluids (urine, serum and cerebrospinal fluid). Although urine is not a conventional sample for $S$. mansoni diagnosis, the authors highlighted that the possibility of detecting the genomic target in this fluid represents an important advance in DNA research because it can provide a non-invasive sample collection, being easily obtainable by the own infected person.

As another alteranative, Sohayeb et al. [32] designed an amperometric immunosensor in which they attached soluble worm antigens (SWA) onto a screen-printed carbon electrode (SPCE). The SWA probes recognized $S$. mansoni antibodies in a buffered solution and the immunocomplex formation was detected by performing differential pulse voltammetry (DPV) in an electrochemical cell composed by the working (functionalized area), counter and reference electrodes of the SPCE and an electrolyte containing $1 \mathrm{M} \mathrm{KCl}$ and $5 \mathrm{mM}\left[\mathrm{Fe}(\mathrm{CN})_{6}\right]^{3-/ 4-}$ redox probe. The cyclic voltammograms were collected in a potential range of $-1.00 \mathrm{~V}$ to $+0.75 \mathrm{~V} v \mathrm{~s} \mathrm{Ag} / \mathrm{AgCl}$ at $50 \mathrm{mV} \cdot \mathrm{s}^{-1} \mathrm{scan}$ rate. In the presence of antibodies, the decrease in the intensity of the redox peak was attributed to the formation of the immunocomplex referring to the detection of the target analyte. The proposed biosensor exhibited a linear response towards the analyte at a concentration range from 0.038 to $20 \mathrm{ng} \cdot \mathrm{mL}^{-1}$.

In 2019, Taman and El-Beshibishi [33] reviewed the status of laboratory diagnosis of schistosomiasis mansoni highlighting the importance of the early diagnosis as a tool to prevent chronic complications and to cease the transmission cycle. The authors mentioned that the current methods are not enough sensitive and mention the detection of new biomarkers (such as Dysferlin and Aquaporin) as a future perspective in this field.

\subsection{Malaria}

Plasmodium falciparum is one of the most important parasites causing infection in humans at high levels of both morbidity and mortality. The intensity of the manifestation of the disease depends on some aspects, such as density, longevity, the biting habits of the mosquito, the victim predisposition (age and whether it is the first bitten or not for instance), etc.. Out of five Plasmodium species, $P$. falciparum infection is responsible for 99\% of deaths and can cause anemia, intrauterine growth retardation and prematurity [34, 35]. The key point of malaria diagnosis lies on the fact that, despite curable, the severity of this disease may progress within up to $24 \mathrm{~h}$ if the appropriate treatment is not managed in time. Furthermore, the appropriate treatment is related to the correct identification of the involved parasite species. To meet those necessities, WHO recommends microscopy or rapid diagnostic tests before initiating the treatment [35]. Accordingly, many researches have been critically devoted to the development of selective and fast diagnostic tests for $P$. falciparum infection. Ragavan et al. [36] listed several disadvantages of the currently available diagnostic assays, several of them similar to those listed herein with respect to other NTDs, such as the costly equipment, the laborious steps for performing the tests, necessity for very skilled staff and lack of onsite applicability.

A research developed in the International Medical University of Kuala Lumpur [37] (Malaysia) reported the fabrication of an optical biosensor capable of providing an efficient and quick detection of the disease in populations from risk locations. Infected rat blood samples were inspected using Ultraviolet-Visible-Near-Infrared (UV-Vis-NIR) spectroscopy during 12 post-infection days. In their results, the authors found a relevant correlation between the spectral absorbance at $650 \mathrm{~nm}$ and the Plasmodium infection from 4 to 7 post-infection days.

As a response to the necessity for fast and in-situ methods for malaria diagnosis, Dutta [30] highlighted the electrochemical biosensors as promising tools. The author reviewed many recent researches at this topic and observed that, amongst many conductive transducer substrates, metallic electrodes provided the best signal-to-noise ratios. Besides, the printing technology have been an important trend in the field of portable biosensors because of the relative 
lower cost compared to other technologies, because it is disposable and offers the possibility of functionalization by several distinct methods $[38,39]$.

Dutta [40] discussed the trends in the development of electrochemical biosensors for diagnosing Malaria. In this work, the author reviewed the recent literature in this field and pointed out that several material have been studied in order to improve the device's performance (indium tin oxide, metallic nanoparticles, graphene, etc.). As outlook, the work brings to our attention the necessity of improving the current technologies with nanomaterials in order to allow, in a close future, the production of point-of-care devices for bedside applications. In order to achieve better conditions for the real application of analytical tools to diagnose Malaria, Paul et al. [41] developed a flexible and lightweight chemiresistive biosensor in a polyethylene terephthalate substrate. The transducer sensitive layer consisted of multiwalled carbon nanotubes, zinc oxide nanofibers and anti-HRP2 antibody. The authors postulated that the changes in the global resistance of the transducer due to the presence of the HRP2 antigen arose from the formation of the immune complex (antibody-antigen). According them, the recognition of the analyte scatters the charge carriers from the $\mathrm{ZnO}$ nanofibers because of the electrostratic gating effect. With a linear response between $10 \mathrm{fg} \cdot \mathrm{mL}^{-1}$ to $10 \mathrm{ng} \cdot \mathrm{mL}^{-1}\left(\mathrm{R}^{2}=\right.$ 0.978), the proposed biosensor presented a LOD equal to $0.97 \mathrm{fg} \cdot \mathrm{mL}^{-1}$ and a sensitivity of $8.29 \mathrm{k} \Omega \cdot \mathrm{g}^{-1} \mathrm{~mL}$.

\subsection{Snakebite envenoming}

Most but not exclusively present in rural areas, snakebite envenoming was removed from the WHO's list of in 2013 but returned to the document as one of the most important NTD in 2017. Snakebite accidents receive a special attention mainly because of the high incidence of the cases and due to their important severity [42]. Chippaux [42] reported that there are some global challenges with respect to the treatment of bitten victims. Amongst these challenges, the author highlights the importance of the rapid administration of the serum in order to succeed the treatment. Furthermore, he reiterates that the serum must be very specific to the venom of the offending snake because it provokes the development of antibody-antigen interactions in the victim's blood causing the neutralization of the venom effect. Accordingly, the accuracy and rapidity of the diagnosis in this case is a key feature that can preserve the life of the victim.

Despite some analytical techniques are already available worldwide, such as the traditional Enzyme-Linked Immunosorbent Assay (ELISA) and horse-peroxidase induced precipitation, these methods need longtime and very costly experimentation to provide the diagnosis [43]. Aiming to overcome these limitations, in 2018, Faria et al. [44] proposed a non-Faradaic impedimetric immunosensor for detecting the venom from Bothrops snakes, the most important snake genus in Brazil with respect to the frequency of envenoming cases. In their findings, the authors reported a LOD equal to $0.27 \mu \mathrm{g} \cdot \mathrm{mL}^{-1}$ with high selectivity towards the bothropic venom. The leakage resistance was monitored as a transduction signal related to the recognition of the venom in buffered solution and the device presented almost no impedimetric signal towards the presence of heterologous venoms (from Crotalus and Micrurus snakes).

Choudhury et al. [45] constructed an antibody-based sensor employing SPR as transduction techniques. In their achievements, the authors demonstrated that this biosensor was capable to detect Indian cobra venom at various concentrations $\left(0.1 \mathrm{mg} \cdot \mathrm{mL}^{-1}\right.$ to $\left.1.0 \mathrm{mg} \cdot \mathrm{mL}^{-1}\right)$ in buffered solution. The detection of the venom antigens red-shifted the resonance dip due to the alteration of the local refractive index as a result of the modification of the sensor surface.

\subsection{Leishmaniasis}

Prevalent in the tropical Africa, Asia and Latin America, Leishmaniasis affects approximately 2 million people each year. The disease is caused by flagellate protozoan of Leishmania spp. and is transmitted by sandflies bite [46]. In a study of endemicity released in 2018 , the WHO reported that leishmaniasis is endemic in 86 countries as the cutaneous form and in 76 countries as visceral leishmaniasis [47]. The former is the most common clinical manifestation in humans, despite the other is the most severe, causing a several number of deaths if untreated. The manifestation of the disease affects not only humans but also domestic mamals, being the dogs the most common reservoir of the parasite in urban regions [48]. Tiburcio et al. [49] mentioned that the mortality data is very scare and mainly reflects the registers of deaths in hospitals. Contrarily, the number of reported cases seems to increase in the last years partially due to the improvements in the development of diagnostic tools.

The work published by Nazari-Vanani et al. [50] showed the development of a genosensor that used chemically modified cadmium sulfide nanosheets (CSN) to capture the genomic DNA of Leishmania infantum. This biosensor 
was produced by means of the deposition of a thin layer of CSN on a Platinum disk electrode through the formation of $\mathrm{Cd}(\mathrm{OH})_{2}$ and immersion of this electrode in a $\mathrm{Na}_{2} \mathrm{~S}$ bath. The developed layer had an average length of $0.4 \mu \mathrm{m}-$ $2.1 \mu \mathrm{m}$ and an average thickness of $67.3 \mathrm{~nm}$. Finally, an oligonucleotide sequence (probe oligonucleotide, PO) from a homology of non-L. infantum was immobilized on the transducer. The tests indicated that the genosensor provided good reproducibility, with a relative standard deviation (RSD) of $6.2 \%$ in the $\mathrm{R}_{\mathrm{ct}}$, sufficient selectivity and a LOD of 1.2 $\mathrm{ng} \cdot \mu \mathrm{L}^{-1}$. The stability of the biosensor was estimated to be approximately 20 days of storage time. After this period, the authors found a decrease in the device performance.

Cordeiro et al. [51] developed an impedimetric immunosensor based on modified screen-printed gold electrodes (SPE-Au) to detect Leishmania infantum. They used samples of the protozoan culture and sera from infected dogs to test the applicability of the produced biosensor. The SPE-Au surface was immobilized with 3-mercaptopropionic acid (MPA), N-hydroxysuccinimide (NHS) and N-(3-dimethylaminopropyl)-N'-ethylcarbodiimide hydrochloride (EDC) in order to form a self-assembled monolayer (SAM). Then, L. infantum antigens were added to the immunosensor at concentrations from 12.5 to $100 \mu \mathrm{g} \cdot \mathrm{mL}^{-1}$. In a next step, positive and negative canine serum samples were prepared at 1:40, 1:80, 1:160, 1:320,1:640 and 1:1280 dilutions. The samples previously went through serological analyzes in order to confirm the presence/concentration of the antigens. Electrochemical tests applied to the SPE-Au, SAM/SPE-Au and antigen/SAM/ SPE-Au electrodes demonstrated that impedance of the immunosensor increased up to the concentration of $50 \mu \mathrm{g} \cdot \mathrm{mL}^{-1}$, when surface saturation occurs. Moreover, since there was a difference between the impedance values presented in positive and negative samples, thus, the immunosensorwas able to test whether a patient is infected or not. The biggest difference between the response analytical data occurred at 1:160 dilution, which was defined as the ideal dilution for carrying out the analyzes [36].

\subsection{Chagas disease}

There are more than 100 years since, in Brazil, the physician Carlos Chagas [52] firstly reported the disease that came to receive his name and, even nowadays, still represents an important global concern to humans' health because of its potential morbidity and mortality. Despite its major presence in Latin America, population migration and some other mechanisms of transmission have posed Chagas disease as a global concern [53]. Nonetheless, once the vectorial route is not the only transmission mechanism (it can be transmitted by mother to unborn child, through oral transmission in contaminated food, medical procedures, etc.), the disease also affects non-endemic countries worldwide [54].

According to WHO [55], more than 6 million people are infected by the parasite in the world and approximately 25 million habit regions considered as vector transmission areas. Besides the healthcare corner, Pérez-Molina and Molina [53] estimated an economic loss of US\$ 1.2 billion in productivity due to premature deaths in the seven southernmost countries in America.

The transmission of Chagas disease, also known as American trypanosomiasis, is mainly caused by blood-sucking triatomine bugs infected with a group of protozoan parasites belonging to Trypanosoma cruzi lineages [55]. Due to the own nature of the disease, its diagnosis has been considered challenging. Chagas disease presents a primary stage called "acute phase" is asymptomatic. At the end of the acute phase, after 4-8 weeks, the person remains infected if untreated and starts to develop the chronic phase. This second stage can last some decades and the parasite is not easily detectable, although it can manifest some chronic infections. As a result, the number of known cases of Chagas disease is considered significantly underestimated $[53,56]$.

The most conventional techniques used to diagnose Chagas disease consists of serological techniques, amongst which ELISA, indirect immunofluorescence and indirect hemagglutination assay are the more frequently employed ones [56]. Despite considered assertive to detect parasite-specific immunoglobulins, these techniques require laborious methods and skilled staff to perform and interpret the tests, which represents an important barrier especially in remote regions where the disease is very common. Mayta et al. [57] also highlight Polymerase Chain Reaction (PCR) as an eminent sensitive analytical tool for this regard. However, the authors mentioned that conventional PCR presents a major disadvantage the possibility of cross-contamination, besides the fact its negative results cannot be considered as infection absence. In order to provide accurate solutions for the diagnosis of Chagas disease, some researchers have devoted their works to the development of biosensors for various techniques.

Theint et al. [58] studied the optical spectrum of murine blood at $695 \mathrm{~nm}$ in pre and post infection by Plasmodium 
berghei and Trypanosoma avensi. Measuring the Ultraviolet-Visible-Near-infrared absorbance of the samples up to the $13^{\text {th }}$ post infection day, the researchers reported significant absorbance differences arising from the Plasmodium infected blood. Contrarily, no significant variation between the basal signal and the spectrum referring to the last day was observed for Trypanosoma infected sample. According to the authors, this difference possibly arose from the different pathogenesis mechanisms of each infecting agent, because while Plasmodium berghei causes the disruption of red blood cells, Trypanosoma acts on the host's tissues and organs, multiplying its trypomastigotes.

Luz et al. [59] employed the SPR technique to detect the presence of antibodies anti-Trypanosoma cruz in human blood with their fabricated biosensor. A total of 57 samples were analyzed, among which 20 arose from non-infected people and the others from individuals previously diagnosed as positives to Chagas disease. Based on their promising results, the authors defined an optimal cut-off signal (displacement of the SPR angle equal to $17.2^{\circ}$ ) to differentiate the analytical response towards chagasic and non-infected individuals. In a second stage, the authors attested their sensor to present minor cross-reactivity when analyzing 42 blood samples of non-chagasic individuals infected with other important diseases: Visceral Leishmaniasis, Cutaneous Leishmaniasis, Acute Toxoplasmosis, Chronic Toxoplasmosis, $\mathrm{T}$ lymphotropic virus, hepatitis B virus and human immunodeficiency virus (HIV). Finally, a comparison between the performances of the authors' biosensor and an ELISA test corroborated that the proposed assay is very competitive and presented practically the same accuracy.

Regiart et al. [60] decorated screen-printed carbon electrodes (SPCEs) with gold nanoparticles and recombinant shed acute phase antigen to detect anti-Trypanosoma cruz Immunoglobulin M (IgM) antibodies. The immune interactions derived from the biosensing phenomenon hindered the electron-transfer processes at the electrodeelectrolyte interface, causing alterations on the global SPCEs current. This amperometric detection led to the linear increasing of the current as a response the increasing analyte concentration (from 10 to $200 \mathrm{ng} \cdot \mathrm{mL}^{-1}$ ). The authors highlighted as an important advantage of their immunological device its capability to detect anti-T. cruzi IgM in newborns, which makes this biosensor capable to distinguish congenitally infected infants from the uninfected ones.

Due to the challenging diagnosis of Chagas disease and the risk it poses to humans' health, Vorobioff's group have contributed to the scientific literature with two works $[61,62]$ related to the development of analytical tools for detecting the presence of the disease transmitters. In both contributions, the authors report the application of electronic noses for detecting volatile secretions of Triatoma infestans, distinguishing between the vectors sexes and their stage of development.

\section{Conclusion}

Due to the high levels of incidence, morbidity and mortality of the NTDs worldwide, a great concern have been devoted to the impact of these diseases and the methods for treating them. As a similar aspect, the early diagnosis represents a key aspect to the efficiency of the treatments. However, the current available assays for detecting the target compounds related to these illnesses present some limitations that hinders the efficiency of the diagnosis. The conventional techniques are generally very time consuming, demand sophisticated and expensive equipment, besides the requirement for very skilled technical staff to carry out the measurements, they are not amenable for in-situ applications and in some cases they not even exhibit enough sensitivity nor selectivity. For most NTDs, PCR and ELISA are classical diagnostic methods, differing from one disease to other about the use of the specific target (DNA strand, antigen and/or antibody). Despite very sensitive, these assays present all the previous mentioned drawbacks.

Accordingly, some researches presented for the detection of Zika virus, dengue, schistosomiasis, malaria, snakebite envenoming, leishmaniasis and Chagas disease have shown new findings to facilitate the treatment and to prevent the clinical worsening of patients. In this sense, we have reported the recent trends in the development of biosensors as an alternative to the limited traditional methods. Published works in the field of biosensing technologies for diagnosing NTDs have presented promising results, indicating low LODs, high selectivity towards the analytes of interest, good stability and low effect of interfering species. Amongst the transduction possibilities, the electrochemical biosensors seem to be more exploited in the most recent publications, achieving attractive sensitivity levels besides their known advantage of being amenable for miniaturization and, consequently, can be used in point-of-care diagnosis. Given the advances in the area of biosensors, the recent academic efforts are expected to converge to the global public concern 
in order to eradicate the NTDs especially in the neediest regions of the planet, bringing more dignity and healthy life expectancy on an equal basis for all populations.

Despite the several advantages in the use of biosensors for diagnosing NTDs, it is worth mentioning that more research is still required to consider this technology for real applications. Even the most recent published literature in this field relies on laboratory-dependent assays, requiring high cost equipment with large dimensions. Thus, considering that the highest incidences of NTDs occur in remote regions, far from the medical centers, suffering from poverty and basic limitations (the access to basic sanitation for instance), we expect that future works will mainly focus on the optimization of biosensors towards their miniaturization to meet requirements for in-situ measurements. Further optimizations concerning the reduction of the response time as well as the automation of analytical responses with algorithms and neural networks are expected to be investigated in the next years to make these devices more user friendly and compatible with current needs.

\section{References}

[1] World Health Organization (WHO). Neglected Tropical Diseases. Available from: https://www.who.int/neglected diseases/diseases/en/ [Acessed 13rd November 2019].

[2] Boisson S, Engels D, Gordon BA, Medlicott KO, Neira MP, Montresor A, Solomon AW, Velleman Y. Water, sanitation and hygiene for accelerating and sustaining progress on neglected tropical diseases: A new global strategy 2015-20. International Health. 2016; 8(1): 19-21.

[3] Machini WBS, Oliveira-Bretti AM. Antileishmanial drug miltefosine-dsDNA interaction in situ evaluation with a DNA-electrochemical biosensor. Electroanalysis. 2018; 30(1): 48-56.

[4] Almeida TSO, Ramalho SNL. Delineamento das doenças tropicais negligenciadas no Brasil e o seu impacto social. Ver InterSci. 2017; 5(2): 69-91.

[5] Vasconcellos VA, Lins VFC, Faria RAD. Application of biosensors in the petrochemical industry: A mini review on the sensing platforms for polycylic aromatic hydrocarbons detection. International Journal of Biosensors \& Bioelectronics. 2019; 5(4): 142-148.

[6] Kaushik A, Yndart A, Kumar S, Jayant RD, Vashist A, Brown AN, Li CZ, Nair M. A sensitive electrochemical immunosensor for label-free detection of Zika-virus protein. Scientific Reports . 2018; 8: 1-5.

[7] Souto DEP, Volpe J, Gonçalves CC, Ramos CHI, Kubota LT. A brief review on the strategy of developing SPRbased biosensors for application to the diagnosis of neglected tropical diseases. Talanta. 2019; 205.

[8] Fiel WA, Borges PAF, Lins VFC, Faria RAD. Recent advances on the electrochemical transduction techniques for the biosensing of pharmaceuticals in aquatic enviroments. International Journal of Biosensors \& Bioelectronics. 2019; 5(4): 119-123.

[9] Shetti NP, Bukkitgar SD, Reddy KR, Reddy CV, Aminabhavi TM. ZnO-based nanostructured electrodes for electrochemical sensors and biosensors in biomedical applications. Biosensors \& Bioelectronics. 2019; 141: 111417.

[10] Shetti NP, Bukkitgar SD, Reddy KR, Reddy CV, Aminabhavi TM. Nanostructured titanium oxide hybrids-based electrochemical biosensors for healthcare applications. Colloids and Surfaces B: Biointerfaces. 2019; 178: 385394.

[11] Faria RAD, Houmard M, Rosário VAM, Lins VFC, Heneine LGD, Matencio T. $\mathrm{TiO}_{2}$ sol-gel coating as a transducer substrate for impedimetric immunosensors. Chemical and Biochemical Engineering Quarterly. 2019; 33(4): 437-447.

[12] World Health Organization (WHO). Working to overcome the global impact of neglected tropical diseases. Available from: https://apps.who.int/iris/bitstream/handle/10665/44440/9789241564090_eng. pdf;jsessionid=C70D87926D2B4E7C143F77FA1DC83AA7?sequence=1 [Acessed 15th November 2019].

[13] Petersen LR, Jamieson DJ, Powers AM, Honein MA. Zika virus. The New England Journal of Medicine. 2016; 374: 1552-1563.

[14] Bailey GP, Rosenberg ES, Doyle K, JM Jordan, GA Santiago, L Jlein, JP Padilla, FA Medina, SH Waterman, LE Adams, MJ Lozier, JB Pasarell. Persistence of Zika virus in body fluids-final report. The New England Journal of Medicine. 2018; 379: 1234-1243.

[15] Faria AMF, Zucolotto V. Label-free electrochemical DNA biosensor for Zika virus identification. Biosensors \& Bioelectronics. 2019; 131: 149-155.

[16] Priye A, Bird SW, Light YK, Ball CS, Negrete OA, Meagher RJ. A smartphone-based diagnostic platform for rapid 
detection of Zika, chikungunya, and dengue viruses. Scientific Reports. 2017; 7: 1-7.

[17] Theel ES, Hata DJ. Diagnostic testing for Zika virus: A postoutbreak update. Journal of Clinical Microbiology. 2018; 56(4): 1-12.

[18] Xavier AR, Kanaan S, Bozzi RP, Amaral LV. Clinical and laboratory diagnosis of Zika fever: An update. Jornal Brasileiro de Patologia e Medicina Laboratorial. 2017; 53(4): 252-257.

[19] Tancharoen C, Sukjee W, Thepparit C, Jaimipuk T, Auewarakul P, Thitithanyanont A, Sangma C. Electrochemical biosensor based on surface imprinting for Zika virus detection in serum. ACS Sensors. 2018; 4: 69-75.

[20] Kim JH, Cho CH, Ryu MY, Kim JG, Lee SJ, Park TJ, Park JP. Development of peptide biosensor for the detection of dengue fever biomarker, nonstructural 1. Plos One. 2019; 14(9): 1-13.

[21] Lanciotti RS, Calisher CH, Gubler DJ, Chang GJ, Vorndam AV. Rapid detection and typing of dengue viruses from clinical samples by using reverse transcriptase-polymerase chain reaction. Journal of Clinical Microbiology. 1992; 30(3): 543-551.

[22] Rodrigues MB, Freire HB, Corrêa PR, Mendonça ML, Silva MR, França EB. Is it possible to identify dengue in children on the basis of Ministry of Health criteria for suspected dengue cases? Jornal de Pediatria. 2005; 81: 209215.

[23] Jahanshahi P, Zalnezhad E, Sekaran SD, Adikan FRM. Rapid immunoglobulin m-based dengue diagnostic test using surface plasmon resonance biosensor. Scientific Reports. 2014; 4: 1-7.

[24] Bassoa CR, Crulhasa BP, Magrob M, Vianellob F, Pedrosa VA. A new immunoassay of hybrid nanomater conjugated to aptamers for the detection of dengue virus. Talanta. 2019; 197: 482-490.

[25] Zainuddin AA, Nordin AN, Asri MAM, Rahim RA, Guines C, Chatras M, Pothier A, Mak WC. Development of integrated electrochemical-quartz crystal microbalance biosensor arrays: towards ultrasensitive, multiplexed and rapid point-of-care Dengue detection. In: 12th International Conference on Biomedical Electronics and Devices. 2019. p. 220-227.

[26] Monsalve CO, González JMG, Grajales MJ. Immobilization of DNA probes on a high frequency piezoelectric biosensor. DYNA. 2020; 87(212): 163-168.

[27] WHO. Schistosomiasis, Number of people treated worldwide in 2014. Weekly Epidemiological Record. 2016; 5(91): 53-60.

[28] Scholte RG, Gosoniu L, Malone JB, Chammartin F, Utzinger J, Vounatsou P. Predictive risk mapping of schistosomiasis in Brazil using Bayesian geostatistical models. Acta Tropica. 2014; 132: 57-63.

[29] Mazigo HD, Heukelbach J. Diagnostic performance of Kato Katz technique and point-of-care circulating cathodic antigen rapid test in diagnosing schistosoma mansoni Infection in HIV-1 co-infected adults on the shoreline of lake victoria, tanzania. Tropical Medicine and Infectious Disease. 2018; 3(54): 1-11.

[30] Wu Y, Liu J, Lin Y, Weng R, Chen R, Li J, Lv Z. Diagnosis, monitroing, and control of schistosomiasis-an update. Journal of Biomedical Nanotechnology. 2018; 14: 1-26.

[31] Santos GS, Caldas RGSC, Melo FL, Bruscky IS, Silva MAL, Wanderley LB, Oliveira MDL. Label-free nanostructured biosensor for Schistosoma mansoni detection in complex biological fluids. Talanta. 2019; 204: 395-401.

[32] Sohayeb M, Arida H, Mersal GAM, El-Badawy M. Development of a nanotechnology-based screen-printed biosensor for detection of schistosoma mansoni antibodies. International Journal of Electrochemical Science. 2016; 11: 1337-1344.

[33] Taman A, El-Behibishi SN. Laboratory diagnosis of schistosomiasis mansoni: Current status and future trends. Asian Pacific Journal of Tropical Medicine. 2019; 12(6): 243-249.

[34] White NJ. Anaemia and malaria. Malaria Journal. 2018; 17: 1-17.

[35] Cheung YW, Dirkzwager RM, Wong WC, Cardoso J, Costa JDN, Tanner JA. Aptamer-mediated plasmodiumspecific diagnosis of malaria. Biochimie. 2017; 1-6.

[36] Ragavan KV, Kumar S, Swaraj S, Neethirajan S. Advances in biosensors and optical assays for diagnosis and detection of malaria. Biosensors \& Bioelectronics. 2018; 105: 188-210.

[37] Theint HT, Walsh JE, Tung WS, Leong KVG, Shitan M. Development of an optical biosensor for the detection of trypanosoma evansi and plasmodium berghei. Spectrochimica Acta Part A: Molecular and Biomolecular Spectroscopy. 2019; 218: 348-358.

[38] Dutta G. Electrochemical biosensors for rapid detection of malaria. Materials Science for Energy Technologies. 2019; $1-31$.

[39] Faria RAD, Messaddeq Y, Heneine LGD, Matencio T. Application of screen-printed carbon electrode as an electrochemical transducer in biosensors. International Journal of Biosensors \& Bioelectronics. 2019; 5(1): 1-2.

[40] Dutta G. Electrochemical biosensors for rapid detection of malaria. Materials Science for Energy Technologies. 
2020; 3: 150-158.

[41] Paul KB, Panigrahi AK, Singh V, Singh SG. Multi-walled carbon nanotubes-zinc oxide nanofiber based flexible chemiresistive biosensor for malaria biomarker detection. Analyst. 2017; 1-7.

[42] Chippaux JP. Snakebite envenomation turns again into a neglected tropical disease! Journal of Venomous Animals and Toxins including Tropical Diseases. 2017; 23-38.

[43] Hartono D, Lai SL, Yang KL, Yung LY. A liquid crystal-based sensor for real-time and label-free identification of phospholipase-like toxins and their inhibitors. Biosensors \& Bioelectronics. 2009; 24: 2289-2293.

[44] Faria RAD, Lins VFC, Nappi GU, Matencio T, Heneine LGD. Development of an impedimetric immunosensor for specific detection of snake venom. BioNanoScience. 2018; 8: 988-996.

[45] Choudhury SN, Konwar B, Kaur S, Doley R, Mondal B. Study on snake venom protein-antibody interaction by surface plasmon resonance spectroscopy. Photonic Sensors. 2018; 8(3): 193-202.

[46] Santos SS, Araújo RV, Giarolla J, Seoud OE, Ferreira EI. Searching for drugs for Chagas disease, leishmaniasis and schistosomiasis: A review. International Journal of Antimicrobial Agents. 2020; 55(4): 105906.

[47] World Health Organization (WHO). Leishmaniasis-Epidemiological situation. Available from: https://www.who. int/leishmaniasis/burden/en/ [Accessed 13rd June 2020].

[48] Alves CJ, Azevedo SS, Santos CSAB, Melo MA, Soares RR, Barnabé NNC, Rodrigues GQ. Clinical aspects and diagnosis of leishmaniasis in equids: A systematic review and meta-analysis. Revista Brasileira de Parasitologia Veterinaria. 2019; 28(4): 574-581.

[49] Tiburcio MGS, Pereira VBR, Ramirez LE. Human leishmaniasis in Brazil: A general review. Revista da Associação Médica Brasileira. 2018; 64(3): 281-289.

[50] Nazari-Vanania R, Helia H, Sattarahmady N. An impedimetric genosensor for leishmania infantum based on electrodeposited cadmium sulfide nanosheets. Talanta. 2020; 217.

[51] Cordeiro TAR, Gonçalves MVC, Franco DL, Reis AB, Martins HR, Ferreira LF. Label-free electrochemical impedance immunosensor based on modified screen-printed gold electrodes for the diagnosis of canine visceral leishmaniasis. Talanta. 2018; 195: 327-332.

[52] Chagas C. Nova tripanozomiaze humana: estudos sobre a morfolojia e o ciclo evolutivo do Schizotrypanum cruzi n. gen., n. sp., ajente etiolojico de nova entidade morbida do homem. Memorias do Instituto Oswaldo Cruz. 1909; 1(2): 159-218.

[53] Pérez-Molina JA, Molina I. Chagas disease. The Lancet. 2018; 391: 10115.

[54] Gaso MIR, Gómez LJV, Beyssen D, Sarry F, Carranza MAR, Cerdeña CNI. Biosensors to diagnose Chagas disease: A review. Sensors. 2017; 17(11): 2629.

[55] World Health Organization (WHO). Chagas disease (also known as American trypanosomiasis). Available from: https://www.who.int/en/news-room/fact-sheets/detail/chagas-disease-(american-trypanosomiasis) [Accessed 13rd June 2020].

[56] Egüez KE, Padilla JA, Terán C, Chipana Z, García W, Torrico F, Gascon J, Beltran DFL, Pinazo MJ. Rapid diagnostic tests duo as alternative to conventional serological assays for conclusive Chagas disease diagnosis. PLoS Neglected Tropical Diseases. 2017; 11(4): e0005501.

[57] Mayta H, Romero YK, Pando A, Verastegui M, Tinajeros F, Bozo R, Frost JH, Colanzi R, Flores J, Lerner R, Bern C, Gilman RH. Improved DNA extraction technique from clot for the diagnosis of Chagas disease. PLoS Neglected Tropical Diseases. 2019; 13(1): e0007024.

[58] Theint HT, Walsh JE, Wong ST, Voon K, Shitan M. Development of an optical biosensor for the detection of trypanosoma evansi and plasmodium berghei. Spectrochimica Acta Part A Molecular and Biomolecular Spectroscopy. 2019; 218: 348-358.

[59] Luz JGG, Souto DEP, Assis GFM, Lana M, Luz RCS, Filho OAM, Damos FS, Martins HR. Applicability of a novel immunoassay based on surface plasmon resonance for the diagnosis of Chagas disease. Clinica Chimica Acta. 2016; 454: 39-45.

[60] Regiart M, Pereira SV, Bertolino FA, Garcia CD, Raba1 J, Aranda PR. An electrochemical immunosensor for anti-T. cruzi IgM antibodies, a biomarker for congenital Chagas disease, using a screen-printed electrode modified with gold nanoparticles and functionalized with shed acute phase antigen. Microchimica Acta. 2016; 183: 1203-1210.

[61] Vorobioff J, Boggio N, Lamagna A, Salomón O, Rinaldi C. Improvement of the electronic nose response to detect presence of transmitters of Chagas disease. 2017 ISOCS/IEEE International Symposium on Olfaction and Electronic Nose (ISOEN). Montreal, QC; 2017. p.1-3.

[62] Vorobioff J, Videla E, Boggio N, Salomón O, Lamagna A, Rinaldi CA. Laser vaporization e-Nose method for the detection of transmitter of Chagas disease. Sensors and Actuators B Chemical. 2018; 257: 200-206. 